\title{
EFFICIENCY AND SAFETY OF LEFT ATRIAL APPENDAGE CLOSURE
}

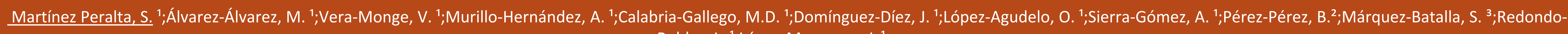
Robles, L. '; López-Mesonero, L. ${ }^{1}$

1Complejo Asistencial Unviersitario de Salamanca, Neurology, Salamanca, Spain

2 Complejo Asistencial de Zamora, Comunity Medicine, Zamora, Spain

3 Complejo Asistencial Unversitario de Salamanca, Radiology, Salamanca, Spain

\section{INTRODUCTION}

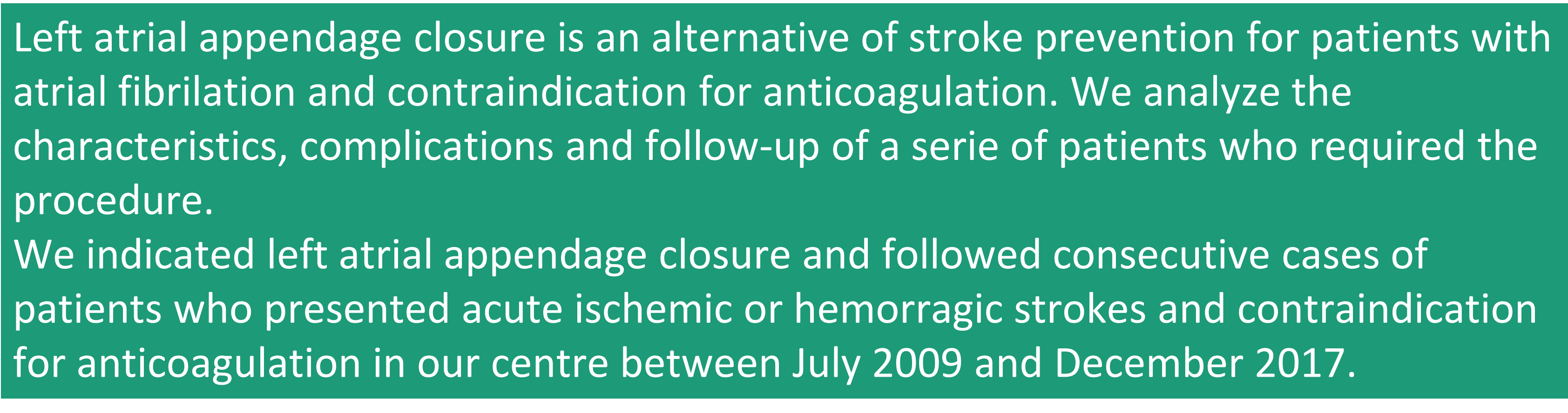

\begin{tabular}{|lc|}
\hline Age & $79 \pm 7$ \\
\hline Gender & \\
\hline Male & $49(50.5)$ \\
\hline Female & $48(49.5)$ \\
\hline CHA2DS2-VASc & $5 \pm 1$ \\
\hline HAS-BLED & $4 \pm 1$ \\
\hline Arterial Hypertension & $87(89.7)$ \\
\hline Mellitus Diabetes & $37(38.1)$ \\
\hline Dyslipidemia & $43(44.3)$ \\
\hline AF & $76(7.2)$ \\
\hline Paroxysmal & $14(14.4)$ \\
\hline Permanent & $66(68)$ \\
\hline Flutter & $5(5.2)$ \\
\hline Stroke & \\
\hline Ischaemic & \\
\hline Hemorragic & \\
\hline Previous treatment & \\
\hline Nother & \\
\hline None & \\
\hline
\end{tabular}

TABLE 1. Basal Characteristics

\begin{tabular}{|ll|}
\hline Follow-up (months) & $23,5[14-39]$ \\
\hline Device-related death & 0 \\
\hline Other-causes death & $10(10)$ \\
\hline Ischemic stroke & $2(2)$ \\
\hline Cortical hemorrage (amyloid angiopathy) & $1(1)$ \\
\hline
\end{tabular}

TABLA 2. Follow-up.

\section{RESULTS}

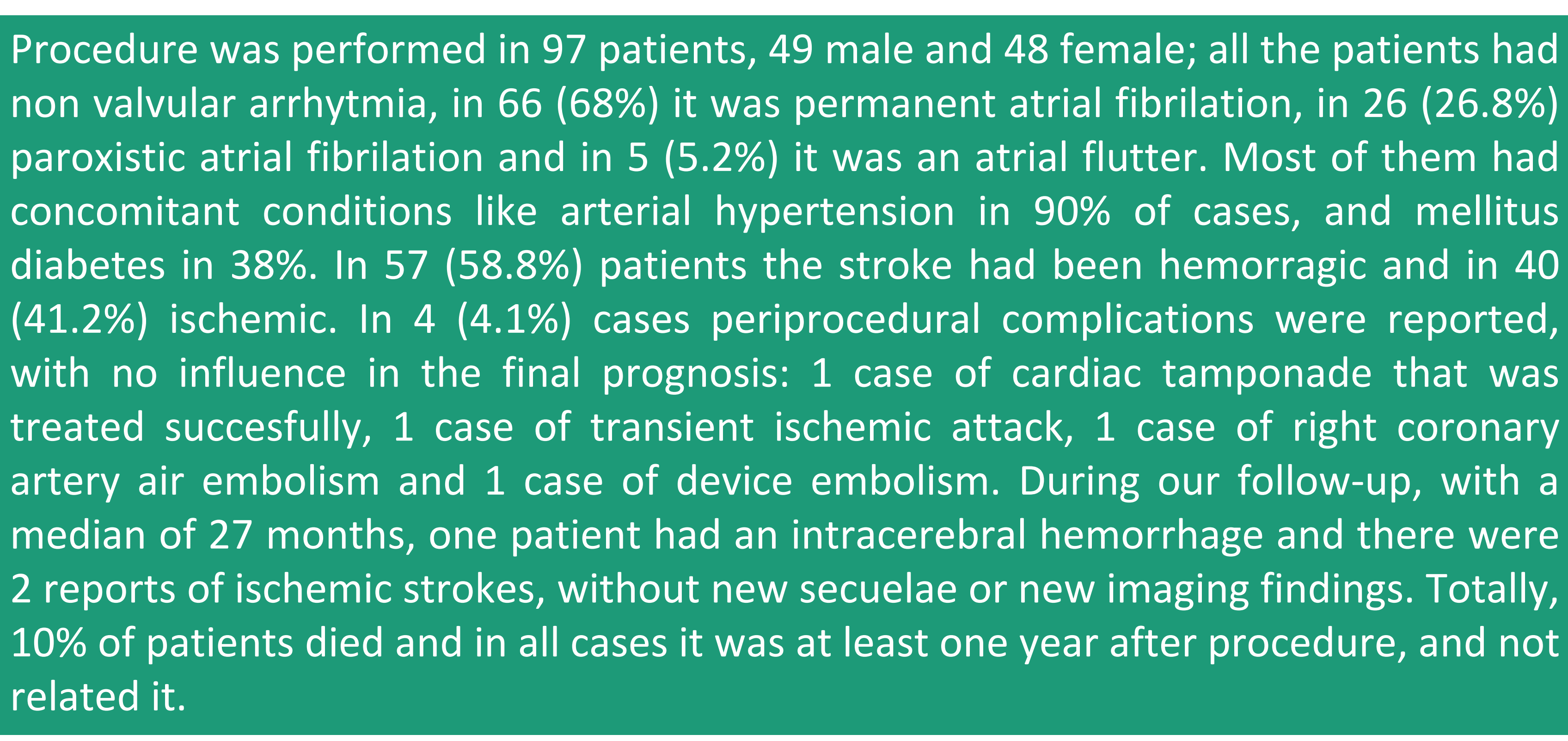

\begin{tabular}{|ll|}
\hline Early LAAC (<3 months after event) & $36(34.6)$ \\
\hline Devices & \\
\hline Amplatzer Cardiac Plug & $4(4.1)$ \\
\hline Amulet & $41(42.3)$ \\
\hline Watchman & $44(45.4)$ \\
\hline LAmbre & $4(4.1)$ \\
\hline Ultraseal & $1(1)$ \\
\hline Periprocedural/inhospital complications & \\
\hline Cardiac tamponade & $1(1)$ \\
\hline Device embolization & $1(1)$ \\
\hline Stroke & 0 \\
\hline TIA & $1(1)$ \\
\hline Coronary artery air embolism & $1(1)$ \\
\hline Death & 0 \\
\hline Discharge treatment & \\
\hline Aspirin + Clopidogrel & $73(75.3)$ \\
\hline LMWH & $6(6.2)$ \\
\hline Aspirin & $7(7.2)$ \\
\hline Clopidogrel & $3(3.1)$ \\
\hline VKA + Aspirin & $3(3.1)$ \\
\hline NOAC & $6(6.2)$ \\
\hline & \\
\hline
\end{tabular}

TABLE 3.Procedure-related characteristics.

Continuous variables are presented as a median [interquartile range] or mean \pm standard deviation. Categorical variables are shown as frequency and percentage. AF: atrial fibrilation. VKA: vitamine $\mathrm{K}$ oral antagonists. NOAC: new oral anticoagulants. LAAC: left atrial appendage closure. TIA: transient ischemic attack. LMWH: low molecular weight heparine.

\section{DISCUSSION}

Left atrial appendage closure can be an efficient and safe alternative for secondary stroke prevention in patients with non valvular atrial fibrilation. According to the data observed in our patient series, this is an intervention with few perioperative complications, low recurrence and low mortality, which is unrelated to the procedure 